\title{
Projeto conceitual de um veículo semiautônomo para sondagem remota de variáveis ambientais
}

\author{
Edel Mary Quinn de Oliveira Figueredo, George Santos Marinho
}

\author{
Programa de Pós Graduação em Engenharia Aeroespacial - Universidade Federal do \\ Rio Grande do Norte (UFRN) - Natal, RN - Brasil \\ edelmaryctegmail.com, gmarinhodct.ufrn.br
}

\begin{abstract}
It presents the conception of a semi-autonomous vehicle for environmental exploration, designed to collect and transmit data on soil variables (moisture and salinity). The project was conceived considering a vehicle capable of moving in areas with potential for desertification, previously identified in satellite images, and helping to characterize environments through data intercomparison. It may also be part of a space system for localized collection, satellite transmission and data reception in a mission control center. From the explorations through the vehicle, the possibility of supporting projects of recovery and soil management, enabling the sustainable use of the soil, is considered.
\end{abstract}

Key-words: Sustainability, Robotic vehicle, Remote sensing.

Resumo. Apresenta-se a concepção de um veículo semiautônomo para exploração ambiental, projetado para coletar e transmitir dados de variáveis do solo (umidade e salinidade). O projeto foi concebido considerando-se um veículo capaz de deslocar-se em áreas com potencial de desertificação, previamente identificadas em imagens de satélites, e auxiliar à caracterização de ambientes por intercomparação de dados. Também poderá fazer parte de um sistema espacial de coleta localizada, transmissão via satélite e recepção de dados em um centro de controle de missão. A partir das explorações por meio do veículo, considera-se a possibilidade de apoio a projetos de recuperação e manejo de solo, viabilizando o uso sustentável do solo.

Palavras-chave: Sustentabilidade, Veículo robótico, Sensoriamento remoto.

Mestrado. Previsão de conclusão: Jan/2022.

\section{Introdução}

A região semiárida do Brasil - considerada a mais povoada das regiões semiáridas da Terra [Correia et al., 2011] — ocupa parte do Nordeste e uma pequena porção do estado de Minas Gerais, correspondendo a uma área de 1,13 milhão de $\mathrm{km}^{2}$, ou 13,25\% do território nacional [Lambais et al., 2019].

Embora seja o semiárido mais chuvoso do planeta [Malvezzi, 2007], possui baixos índices pluviométricos, proporcionando vários meses do ano sem chuvas que, quando ocorrem, são irregularmente distribuídas, resultando em baixa umidade do ar e longos períodos de seca. O elevado índice de radiação solar característico da região, por 
sua vez, ocasiona altas temperaturas do solo e, portanto, baixa retenção de umidade [Correia et al., 2011], tornando-o pedregoso - tipo de solo prevalente [Lucena et al., 2021], de baixa capacidade produtiva e, em geral, com altos índices de salinização.

Ecossistema predominante do semiárido e exclusivo do Brasil, a Caatinga é composta por vegetação de mesmo nome [Lucena et al., 2021]. Ainda pouco notada no que diz respeito à necessidade de preservação e conservação, possui apenas $2 \%$ de sua área preservada [Oliveira et al., 2019].

Terceiro bioma mais degradado e mais ameaçado do Brasil [Correia et al., 2011], a Caatinga abriga a maior população rural do país [Lucena et al., 2021], que desenvolve atividades econômicas associadas ao extrativismo vegetal, agropecuária e agricultura de subsistência interligada à agricultura familiar e à agricultura irrigada. $\mathrm{Na}$ forma como se desenvolvem, as atividades assolam diretamente os poucos recursos hídricos ainda existentes e exaurem o solo já pobre, que sofre desmatamento intenso, originando um processo contínuo de desertificação da região.

A desertificação é identificada como um dos maiores problemas ambientais em escala global, afetando direta e indiretamente mais de um bilhão de pessoas no planeta [Tavares et al., 2019]. A Convenção das Nações Unidas de Combate à Desertificação [UNCCD, 2017] caracteriza-a como uma degradação de terras nas zonas áridas, semiáridas e subúmidas secas do planeta, decorrente tanto de fatores naturais como de ações antrópicas. No Brasil, as áreas suscetíveis à desertificação concentram-se no Semiárido [Accioly et al., 2019], apresentando cerca de $9 \%$ de sua área com alto índice de desertificação, $85 \%$ com índice moderado e $6 \%$ com baixo índice ou ausência de desertificação [Perez-Marin et al., 2020].

O desafio de conter o avanço da desertificação, concomitante à garantia de sobrevivência dos habitantes das regiões ameaçadas, somente será suplantado por meio de vontade, tempo e recursos. Combinar preservação e desenvolvimento sustentável requer: estudo, planejamento e avaliação. Para tanto, técnicas de monitoramento ambiental são imprescindíveis e precedem quaisquer tomadas de decisões.

Entre as técnicas atualmente disponíveis para estudo do ambiente, citam-se: estações de monitoramento fixas, coleta de amostras de solo, sistemas embarcados em balões, aeronaves e foguetes de sondagem, e satélites de monitoramento / mapeamento. Por si só, a disponibilidade dessas técnicas não resolve o problema, constituindo apenas uma ferramenta para se atingir objetivos.

Para que a coleta de dados ambientais seja representativa, não basta dispor de ferramentas de monitoramento - que são, em geral, caras. É necessário investir em recursos humanos habilitados à operação das ferramentas. Entretanto, dispor de recursos materiais modernos e de pessoal altamente qualificado pode, em alguns casos, ser insuficiente para resolver o binômio preservação-desenvolvimento.

Raramente os resultados dos estudos e projetos de investigação do bioma Caatinga chegam ao público-alvo - constituído pela população rural do Brasil, concentrada majoritariamente no semiárido. Consequentemente, há o risco de segregação de beneficiários, visto que a população rural sofre com altos índices de vulnerabilidade social e econômica, destacando-se os baixos níveis de escolaridade, renda e produtividade [Araujo et al., 2020; Barros \& Pordeu, 2017; Pimentel, 2017]. 
Diante do exposto, idealizou-se uma ferramenta de levantamento de variáveis ambientais, na forma de um veículo de exploração remota de regiões semiáridas da Terra, capaz de coletar e transmitir dados de umidade e salinidade do solo.

Entre os critérios adotados na concepção do veículo explorador, destacam-se: baixos custos de operação e manutenção, portabilidade (pequeno porte), integração a um sistema espacial - permitindo transmitir dados via satélite até uma estação de recepção, que também pode servir como centro de controle de missão, capacidade para operar de modo semiautônomo (demanda mínima de recursos humanos); capacidade para cobrir grandes extensões de área em curto espaço de tempo (tanto em propriedades rurais bem como áreas recuperáveis do Semiárido). Acredita-se que, ao ser concebido a partir desses parâmetros, o projeto do veículo será técnica e economicamente viável, com possibilidades de beneficiar número expressivo de habitantes da região semiárida.

\section{Importância da sondagem remota}

O sensoriamento remoto espacial permite monitorar áreas extensas, viabilizando medição de variáveis ambientais de regiões específicas, permitindo, por exemplo, ações de prevenção de acidentes naturais ou antrópicos. Nesse sentido, o monitoramento / geoprocessamento a partir de imagens de satélites também é utilizado para detecção de áreas com potencial de desertificação. Um dos objetivos dos analisadores é identificar diferentes níveis de salinidade e sodicidade do solo [Bouaziz et al., 2011].

A reflectância espectral da luz varia em função da quantidade e do tipo de sal presente no solo. Esse fenômeno constitui a base da técnica de detecção de salinidade por meio de satélites em órbita da Terra. Contudo, a análise da salinidade em grandes áreas apresenta desvantagens quando comparada às medições em áreas pontuais. Nesse caso, é recomendada a medição combinada, onde são considerados dados de sensoriamento e de medições geoquímicas (realizada por meio de sensores instalados diretamente no solo ou por análise de amostra de solo), seguida da intercomparação de resultados [Bouaziz et al., 2011; Fernandes et al., 2020; Oliveira et al., 2018].

Medições in loco acabam sendo pontuais, limitando a área de exploração devido à dificuldade de abranger maiores extensões em um determinado intervalo de tempo com os recursos financeiros e humanos necessários. Para melhorar a coleta de dados de superfície e fomentar a intercomparação com dados de geoprocessamento espacial, pode-se recorrer a um veículo semiautônomo de exploração remota.

O monitoramento móvel pode suprir demandas não atendidas por meio de estações fixas. Um veículo de exploração pode coletar dados de salinidade do solo em tempo real, de forma rápida e cobrir extensa área de coleta. Os dados podem compor um registro, armazenado no veículo ou transmitido para satélites e retransmitido ao centro de controle, possibilitando comparação com dados de geoprocessamento por satélites, de forma a estabelecer um mapa da desertificação em larga escala e com resultados mais robustos. Esse recurso tem sido utilizado para estudos da Terra, da Lua e de Marte. 


\section{Caracterização do veículo para coleta de dados}

Entre as vantagens do uso de um veículo para exploração remota destacam-se: possibilidade de acesso a regiões com condições ambientais extremas, eliminação de riscos envolvendo deslocamento de pessoal a campo e, consequentemente, menor custo.

Para este projeto, o veículo funcionará de forma semiautônoma, operando em ambiente aberto, sujeito a pequenas inclinações de terreno, podendo encontrar regiões mais pedregosas e erosivas. O veículo operará em regiões onde há potencial de desertificação, então a vegetação será pouca ou inexistente, diminuindo a quantidade de obstáculos que o veículo possa estar sujeito.

O veículo deve possuir um sistema mecânico composto por rodas, suspensão e transmissão próprias para atravessar terrenos com relevo acidentado, mantendo estabilidade e tração adequadas. As rodas devem possuir diâmetro e peso que suportem o torque disponível, dotadas de ranhuras para auxiliar a vencer obstáculos, evitando deslizar ou patinar. A transmissão deve resistir às cargas elevadas, em qualquer direção, enquanto o veículo ultrapassa os obstáculos. O chassi deve ser projetado para resguardar os sistemas de controle, energia e de coleta e transmissão de dados, protegendo-os das intempéries que caracterizam o semiárido. Além desses requisitos, deve ser leve, compacto e eficiente, apresentando baixo consumo de energia.

A energia para alimentar o veículo pode ser obtida por meio de painéis solares, devendo ser acoplados a uma bateria elétrica para armazenagem em quantidade suficiente para garantir autonomia para funcionamento noturno.

O sistema de comunicação (i.e., controle e coleta / transmissão de dados) pode ser projetado para proporcionar a interação do veículo com satélites em órbita da Terra e com um centro de controle de missão. As tarefas podem ser pré-definidas, comportando navegação e decisão de rota a partir da visualização de caminho, desvio de obstáculos, coleta e armazenamento de dados. Para isso, podem ser utilizados sensores, como: hodômetros, sonares, câmeras de navegação e medidores de energia.

A coleta de dados de solo (salinidade e umidade) será realizado por meio de uma sonda acoplada ao veículo. A inserção da sonda no solo pode ser feita por meio de uma broca elétrica, controlada pelo computador de bordo.

\section{Metodologia}

Nesta seção descrevem-se detalhes dos sistemas mecânico, eletrônico e de controle, bem como dos sensores, dispositivo de perfuração e outros componentes necessários ao funcionamento de veículo de exploração. Este projeto é de cunho conceitual e contempla análise e identificação das necessidades do projeto - requisitos e objetivos principais, especificações do veículo para que ele seja minimamente viável e uma definição preliminar do projeto.

\subsection{Sistema mecânico e estrutural}

O sistema mecânico é composto de chassi, suspensão, transmissão e rodas. O chassi pode ser feito de alumínio, devido à leveza, resistência e custo acessível [Sancon et al., 2010]. Para evitar sobreaquecimento interno, o chassi pode ser recoberto por tinta refletiva dos raios solares. Prevê-se uma estrutura em formato retangular, com as seguintes dimensões: $350 \mathrm{~mm}$ de comprimento, $250 \mathrm{~mm}$ de largura, $180 \mathrm{~mm}$ de altura e 
espessura de paredes com $4 \mathrm{~mm}$. Os sensores de navegação podem ser encaixados em aberturas localizadas nas paredes frontal e traseira. Uma abertura na base do chassi pode ser feita para passagem da sonda (dispositivo de perfuração e inserção de sensores no solo). Outras aberturas podem ser feitas para encaixar os sistemas de suspensão e transmissão. $\mathrm{O}$ painel solar deve ser acoplado à parte superior do veículo, servindo, a um só tempo, para captação de energia e sombreamento do veículo, com um espaço de cerca de $20 \mathrm{~mm}$ entre as duas superfícies para permitir a circulação de ar.

A mobilidade do veículo pode ser dada por meio de um mecanismo diferencial, combinando compacidade, leveza, estabilidade e poder de tração. A tração pode ser obtida por motores, um para cada eixo, com funcionamento independente, montados lado a lado e conectados aos eixos correspondentes por meio de um diferencial de engrenagem passiva. A transmissão pode ser composta por dois eixos angulares, com engrenagens cônicas nas extremidades para direcionar o torque ao conjunto de rodas. Prevê-se uma saída dupla de engrenagens acopladas ao motor, para proporcionar melhor distribuição de força, inversão do movimento e torque potente nas rodas. A suspensão pode ter perfil pequeno, possibilitando manter distância entre o corpo do veículo e o solo [Canfield et al., 2018; Lv et al., 2019; Roman, 2005].

\subsection{Sistema de energia}

A energia para fazer funcionar o veículo pode ser provida por painéis solares, que alimentarão uma bateria de $12 \mathrm{~V}$. Essa, por sua vez, deve ter capacidade para manter o veículo em funcionamento contínuo. Além de suprir a demanda dos dois servomotores de acionamento dos eixos das rodas, deverá ser garantida energia para alimentar o servomotor de acionamento do dispositivo de perfuração [Fofilos et al., 2014; Roman, 2005; Santos, 2015].

\subsection{Sistema de navegação}

O sistema de navegação fornece os meios com o qual o veículo irá mover-se pelo ambiente de um destino a outro de forma segura, desviando dos obstáculos, realizando suas tarefas pré-programadas e interagindo com o ambiente através de seu sensoriamento e suas ações.

A arquitetura de controle será composta por módulos: de percepção e atuação, de mapeamento e localização, de comportamento reativo e deliberativo e um para coleta e transmissão de dados. Esses módulos serão responsáveis pelo sensoriamento, mapeamento e localização, navegação e comunicação, coleta e transmissão de dados via satélite. Os sensores que irão compor o sistema perceptivo do veículo serão: 2 encoders (um para cada par de roda), 3 sensores ultrassônicos (para cobrir toda a parte frontal do veículos) e 1 módulo GPS.

O sistema apresentará um controle híbrido, baseado em controle deliberativo e reativo, disposto na arquitetura de subsunção, planejando uma tarefa a ser executada a partir de sua prioridade. O veículo utilizará o SLAM para localização e mapeamento simultâneos, construindo um mapa a partir de características topológicas (em sua maioria) e métricas, realizando a previsão e correção de posição a partir do Filtro de Kalman Estendido (FKE).

Inicialmente, serão previamente identificadas áreas em potenciais de desertificação dispostas em meios responsáveis pelo geoprocessamento de imagens de 
satélites, extraindo delas as coordenadas georreferenciais de latitude e longitude. A partir daí tem-se uma área definida, que será subdividida em áreas menores para facilitar no mapeamento da região, devido os requisitos operacionais do sistema.

Será definida uma posição inicial para o módulo de mapeamento e localização global do robô - atuando em conjunto com o GPS, responsáveis pela construção e atualização do mapa. Paralelamente, os sensores farão reconhecimento do ambiente para auxiliar no mapeamento.

Durante a navegação do veículo, os controles reativos e deliberativos vão atuando e se planejando, passando os comandos pros atuadores de acordo com o comportamento sensorial. Será definida uma função de posição para que o veículo possa percorrer toda a região definida, realizando as medições de solo de forma estratégica.

O mapa topográfico será grafado através do ponto inicial e dos pontos no qual o veículo parará para realizar a coleta de variáveis do solo - mostrando a trajetória percorrida pelo veículo entre as marcações. Da parte métrica, serão representados mapas geométricos para identificar os obstáculos presentes na trajetória do veículo. O FKE fará a estimação da posição do veículo para que ele mantenha sua posição, a partir de dados dos encoders.

\subsection{Sistema de sondagem do solo}

O sistema de sondagem do solo é composto por: dispositivo de perfuração, sensor de salinidade e sensor de umidade.

Para perfuração do solo pode-se optar por um fuso trapezoidal, fixado a um mecanismo de uma furadeira portátil, onde um motor de passo acoplado a guias pode ser usado para controlar os movimentos de descida e subida do fuso.

Os sensores são, basicamente, eletrodos de metal que permitem inserção no solo para medição da condutividade e resistividade do solo, determinadas a partir do potencial salino e da quantidade de água. Sugere-se escolher sensores que combinem custo acessível, facilidade de instalação, durabilidade e confiabilidade.

\subsection{Sistema de controle e comunicação}

O computador de bordo pode ser uma placa de circuito impresso, modelo RaspberryPi 3 Modelo A+, SoC Broadcom BCM2837B0 64 bits, com processador ARMv8 1,4 GHz de quatro núcleos, unidade de processamento gráfico dedicada e $512 \mathrm{MB}$ de memória RAM, por se tratar de uma placa de baixo custo, com processamento e memória suficientes (integrados ao processador) para execução dos algoritmos de navegação e controle dos periféricos [Santos, 2015]. Essa unidade de processamento terá capacidade para leitura dos sensores de navegação, envio de sinais de comando aos sistemas de tração, perfuração do solo e processamento dos sinais dos sensores de variáveis ambientais, além de armazenar e transmitir os dados coletados.

Para transmissão e coleta de dados poderão ser utilizados sistemas desenvolvidos no Brasil - e.g., sistema Samanaú $T X$, que opera numa faixa de frequência de 401,6 MHz, permitindo enviar dados aos satélites do Sistema Brasileiro de Coleta de Dados Ambientais (SBCDA), do tipo ARGOS/SDC, que permite adaptação a outros sistemas de coleta de dados [Vitorino et al., 2018]. 
Prevê-se o uso dos satélites SCD1, SCD2 e CBERS-4, do segmento espacial brasileiro, para captação dos dados transmitidos pelo veículo de exploração. Os dados podem ser transmitidos às estações receptoras de Cuiabá (MT) e Alcântara (MA) e, a em seguida, retransmitidos ao Centro de Controle da Missão, situado em Natal (RN), onde serão tratados e armazenados em arquivo para consulta pelos usuários finais [Queiroz et al., 2018; Vitorino et al., 2018].

\section{Resultados esperados}

O veículo de exploração permitirá preencher algumas lacunas decorrentes das limitações dos sistemas fixos de monitoramento. Terá capacidade para operar no Semiárido brasileiro - assim como em qualquer região semiárida da Terra. Servirá para coletar e transmitir dados de umidade e salinidade do solo, podendo ser dotado de sensores de temperatura do solo, umidade do ar e luminosidade (necessários à estimativa de evapotranspiração). Demandará para sua operação apenas uma pequena equipe, visto que funcionará de forma semiautônoma. Terá capacidade parra cobrir grande extensões de área em curto espaço de tempo, tanto em propriedades rurais quanto em áreas sujeitas a processos de desertificação. Deverá, obrigatoriamente, atender gratuitamente ao produtor rural. Deverá fomentar um banco de dados que subsidiará projetos de recuperação de solos degradados e em processo de desertificação, apoiando a exploração sustentável.

No momento estão sendo definidas as características do dispositivo de perfuração, para dar sequência a sua modelagem, simulação e mecanismo de atuação.

\section{Agradecimentos}

Ao Programa de Pós-Graduação em Engenharia Espacial da Universidade Federal do Rio Grande do Norte - UFRN, pelo apoio à pesquisa.

\section{Referências}

Accioly, L. J. de O.; Silva, A. B. da; Angelotti, F. et al. (2019) Mudanças climáticas e desertificação. In: Ximenes, L. F.; Silva, M. S. L. da; Brito, L. T. de L. (Ed). "Tecnologias de convivência com o Semiárido brasileiro". Fortaleza: Banco Nordeste do Brasil, 2019. Cap. 5. p. 161-186.

Araújo, R. L. M. de S.; Araujo, E. M. de; Silva, H. P. da. et al. (2020) Condições de vida, saúde e morbidade de comunidades quilombolas do semiárido baiano, brasil. Revista Baiana de Saúde Pública, Salvador, v. 43, n. 1, p. 226-246, 20 out. 2020. Secretaria da Saúde do Estado da Bahia. http://dx.doi.org/10.22278/23182660.2019.v43.n1.a2988.

Barros, J. D. de S.; Pordeu, A. V. (2017) "Condições socioeconômicas de agricultores residentes em comunidades rurais no município de Cajazeiras, estado da Paraíba". Anais CONIDIS. Campina Grande: Realize Editora, 2017. http://www.editorarealize.com.br/editora/anais/conidis/2017/TRABALHO EV074 MD1 SA2 ID54 07032017144550.pdf, abr. 2021.

Bouaziz, M.; Matschullat, J.; Gloaguen, R. (2011) Improved remote sensing detection of soil salinity from a semi-arid climate in Northeast Brazil. Comptes Rendus 
Geoscience, Paris, v. 343, n. 11-12, p. 795-803, nov. 2011. Elsevier BV. http://dx.doi.org/10.1016/j.crte.2011.09.003.

Canfield, S. L.; Hill, T. W.; Zuccaro, S. G. (2018) Prediction and Experimental Validation of Power Consumption of Skid-Steer Mobile Robots in Manufacturing Environments. Journal Of Intelligent \& Robotic Systems, v. 94, n. 3-4, p. 825-839, 29 mar. 2018. Springer Science and Business Media LLC. http://dx.doi.org/10.1007/s10846-018-0779-7.

Correia, R. C.; Kiill, L. H. P.; Moura, M. S. B. de. et al. (2011) A região semiárida brasileira. In: Voltolini, T. V. (Ed.). Produção de caprinos e ovinos no Semiárido. Petrolina: Embrapa Semiárido, 2011. Cap. 1. p. 21-48. Disponível em: https://ainfo.cnptia.embrapa.br/digital/bitstream/item/54762/1/01-A-regiaosemiarida-brasileira.pdf-, nov. 2020.

Fernandes, A. C. G.; Cavalcanti, L. F. M.; Martins, W. A. et al. (2020) Ferramentas de identificação de salinidade do solo: um estudo acerca das técnicas eficazes no manejo agrícola. GeoGraphos [En línea]. Alicante: Grupo Interdisciplinario de studios críticos y de América Latina (GIECRYAL) de la Universidad de Alicante, 2 de junio de 2020, vol. 11, no 127 p. 133-157. ISSN: 2173-1276. DL: A 371-2013. DOI: 10.14198/GEOGRA2020.11.127.

Fofilos, P.; Xanthopoulos K. I.; Romanos, E. A.; Zikidis, K.; Kanellopoulos, N. (2014) Kerveros I: An Unmanned Ground Vehicle for Remote-Controlled Surveillance. Journal of Computations \& Modelling, v. 4, n. 1, p. 223-236, 2014. ISSN: 17928850 .

Lambais, G. R.; Gomes, V. dos S.; Van Haandel, A. C. et al. (2019) Águas residuárias na produção de mudas florestais do bioma Caatinga. O Semiárido Brasileiro e Suas Especificidades, p. 1-9, 5 ago. 2019. Atena Editora. http://dx.doi.org/10.22533/at.ed.1051905081.

Lucena, R. L.; Ferrer, E.; Guilhermino, M. M. (2021) Mitigando os riscos da seca através de ações de recuperação e preservação do bioma caatinga no semiárido brasileiro. Brazilian Journal Of Development, Curitiba, v. 7, n. 4, p. 36546-36557, abr. 2021. DOI: 10.34117/bjdv7n4-221.

Lv, W.; Kang, Y.; Qin, J. (2019) Indoor Localization for Skid-Steering Mobile Robot by Fusing Encoder, Gyroscope, and Magnetometer. Ieee Transactions On Systems, Man, And Cybernetics: Systems, v. 49, n. 6, p. 1241-1253, jun. 2019. Institute of Electrical and Electronics Engineers http://dx.doi.org/10.1109/tsmc.2017.2701353.

Malvezzi, R. (2007) "Semi-árido: uma visão holística”. Brasília: Confea, 2007. 140 p. https://www.agrisustentavel.com/doc/ebooks/semiarido.pdf, jul. 2021.

Oliveira, C. D. L.; Silva, A. P. A.; Moura, P. A. G. (2019) Distribution and Importance of Conservation Units in the Caatinga Domain. Anuário do Instituto de Geociencias Ufrj, v. 42, n. 1, p. 425-429, 13 maio 2019. Instituto de Geociencias - UFRJ. http://dx.doi.org/10.11137/2019 $1425 \quad 429$.

Oliveira, W. S. N.; Lima, A. S.; Ribeiro, R. B. S. (2018) Identificação de possíveis áreas salinizadas no perímetro irrigado de São Gonçalo utilizando o sensoriamento remoto. 
Revista Ibero Americana de Ciências Ambientais, v.9, n.4, p.362-378, 2018. DOI: http://doi.org/10.6008/CBPC2179-6858.2018.004.0029.

Perez-Marin, A. M.; Araujo, H. A. Q. de; Vendruscolo, J. et al. (2020) Níveis atuais da desertificação no Semiárido brasileiro. In: Congresso Brasileiro De Agroecologia, 11., 2020, São Cristóvão. Anais [...]. São Cristóvão: Associação Brasileira de Agroecologia, 2020. v. 15, n. 2.

Pimentel, N. G. L. (2017) "Levantamento da composição florística nos quintais agroflorestais rurais na região de Mossoró-RN e situação socioeconômica das famílias". 2017. 40 f. TCC (Graduação) - Curso de Engenharia Florestal, Universidade Federal Rural do Semi-Árido, Mossoró, 2017.

Queiroz, K. P.; Dias, S. M.; Duarte, J. M.; Carvalho, M. M. (2018) Uma solução para o Sistema Brasileiro de Coleta de Dados Ambientais baseada em nanossatélites. Holos, v. 7, p. 132-142, 22 dez. 2018. Instituto Federal de Educacao, Ciencia e Tecnologia do Rio Grande do Norte (IFRN). http://dx.doi.org/10.15628/holos.2018.6307.

Roman, M. J. "Design and analysis of a four wheeled planetary rover". (2005) $129 \mathrm{f}$. Tese (Mestrado) - School Of Aerospace And Mechanical Engineering, University Of Oklahoma, Norman, Oklahoma, 2005.

Saccon, V. T.; Parra, B. S.; Olea, C. W.; Santos, J. F. dos; Alcântara, N. G. de. (2010) Caracterização microestrutural e comportamento mecânico das ligas de alumínio AA2139 T3 e T8 soldadas por fricção rotativa com mistura. Soldagem \& Inspeção, v. 15, n. 4, p. 289-297, dez. 2010. FapUNIFESP (SciELO). http://dx.doi.org/10.1590/s0104-92242010000400006.

Santos, P. H. M. (2015) "Desenvolvimento de um robô móvel autônomo de alta velocidade”. 2015. 65 f. TCC (Graduação) - Curso de Engenharia Elétrica, Universidade de São Paulo, São Carlos, 2015.

Tavares, V. C.; Arruda, I. R. P. de; Silva, D. G. da. (2019) Desertificação, mudanças climáticas e secas no semiárido brasileiro: uma revisão bibliográfica. Geosul, Florianópolis, v. 34, n. 70, p. 385-405, jan./abr. 2019. Universidade Federal de Santa Catarina (UFSC). http://dx.doi.org/10.5007/2177-5230.2019v34n70p385.

United Nations Conventions to Combat Desertification (UNCCD). (2017) Convention Text. 2017. https://www.unccd.int/sites/default/files/relevant-links/201701/UNCCD_Convention_ENG_0.pdf, abr. 2021.

Vitorino, B. A. F.; Firmo Filho, A. S.; Lima, L. B. de M.; Souto, M. C. de B.; Carvalho, M. J. M. de. (2018) "Samanaú.TX - Low cost data collect transmitter". https://www.unoosa.org/documents/pdf/psa/activities/2018/Symposium_Brazil_BST I/presentations/poster/P63_Vitorino_Bruno.pdf, jul. 2021. 